\title{
Perfil sensorial e aceitabilidade de méis de abelhas sem ferrão submetidos a processos de conservação
}

\author{
Sensorial profile and acceptability of stingless bee honey submitted to conservation processes
}

Geni da Silva SODRÉ ${ }^{1 \star}$, Carlos Alfredo Lopes de CARVALHO ${ }^{1}$, Antonio Augusto Oliveira FONSECA ${ }^{1}$, Rogério Marcos de Oliveira ALVES ${ }^{1}$, Bruno de Almeida SOUZA ${ }^{2}$

\begin{abstract}
Resumo
O presente trabalho avaliou o perfil sensorial e a aceitabilidade de méis de abelhas sem ferrão submetidos a dois processos de conservação, objetivando obter maior vida de prateleira. Foram utilizadas amostras de méis de Melipona scutellaris e M. quadrifasciata, coletadas no Estado da Bahia entre dezembro de 2005 e janeiro de 2006. As amostras foram submetidas aos processos de pasteurização e desumidificação, passando em seguida por avaliação sensorial. O perfil sensorial foi determinado no Laboratório de Entomologia do Centro de Ciências Agrárias, Ambientais e Biológicas da Universidade Federal do Recôncavo da Bahia, em Cruz das Almas, Estado da Bahia. Os atributos analisados foram: fluidez, cor, aroma, cristalização, sabor e aceitabilidade. Os resultados mostraram que os processos de conservação utilizados não interferem no perfil sensorial e na aceitabilidade do produto.
\end{abstract}

Palavras-chave: conservação do mel; meliponíneos; uruçu; mandaçaia.

\begin{abstract}
The present work was conducted to evaluate the sensorial profile and the acceptability of the honey of stingless bees submitted to two conservation processes seeking to obtain longer shelf life. The samples of Melipona scutellaris and M. quadrifasciata honey were collected in the State of Bahia, Brazil, between December 2005 and January 2006. The samples were first submitted to pasteurization and dehumidification processes and then to sensorial evaluation. The sensorial profile was determined in the Laboratory of Entomology in the Center for Agrarian, Biological, and Environmental Sciences of the Federal University of Recôncavo Bahiano, in Cruz das Almas, in the state of Bahia. The analyzed attributes were: fluidity, color, scent, crystallization, flavour, and acceptability. The results show that the conservation processes used do not interfere with the sensorial profile and the product acceptability.

Keywords: honey conservation; meliponids; uruçu; mandaçaia.
\end{abstract}

\section{Introdução}

O mel das abelhas sem ferrão é muito procurado pelos consumidores, principalmente pelos efeitos terapêuticos que lhe são atribuídos. Contudo, os meliponicultores têm demonstrado preocupação com a conservação da qualidade desse produto, em função do seu elevado teor de umidade, o que favorece o processo de fermentação e promove a sua deterioração.

Resultados de análises de amostras de mel de abelhas sem ferrão revelam valores de umidade entre 16,72 e 45\% (CARVALHO et al., 2005; FONSECA et al., 2006). Considerando que o limite máximo para a umidade deve ser igual a $20 \%$, acima do qual o mel estará sujeito à fermentação (FRÍAS; HARDISSON, 1992), a maioria dos méis dessas abelhas está suscetível à contaminação por microrganismos.

Diversas técnicas são empregadas para conservação de alimentos, dentre as quais a desumidificação, a pasteurização e a refrigeração, que têm por objetivo principal manter durante o maior espaço de tempo possível suas qualidades sanitárias, organolépticas e nutricionais (SILVA, 2000).
Métodos analíticos/instrumentais efetivos em detectar o surgimento de problemas durante o processo de conservação de alimentos são conhecidos na literatura, embora muitas vezes se tornem incapazes de medir alterações perceptíveis que afetam a aceitação do produto (CARDELLO; CARDELLO, 1998).

A análise sensorial é uma técnica de avaliação de atributos perceptíveis pelos órgãos do sentido (atributos organolépticos) e é usada em muitos campos, permitindo estabelecer o perfil organoléptico de diversos produtos, indicando a preferência do consumidor (PIANA et al., 2004). As características sensoriais estimulam os sentidos e provocam vários graus de reações de desejo ou rejeição, em que o consumidor escolhe um alimento pelo seu nível de qualidade sensorial (ARAÚJO et al., 2000).

De acordo com Carvalho et al. (2006), os atributos sensoriais do mel de abelhas sem ferrão, como aroma, sabor, cor, fluidez e cristalização, fornecem informações relevantes para a identificação e promoção desse produto, agregando valor que é convertido em renda para os meliponicultores.

Recebido para publicação em 16/4/2007

Aceito para publicação em 17/7/2008 (002465)

${ }^{1}$ Grupo de Pesquisa Insecta, Centro de Ciências Agrárias, Ambientais e Biológicas, Universidade Federal do Recôncavo da Bahia - UFRB, CEP 44380-000,

Cruz das Almas - BA, Brasil, E-mail: gssodre@yahoo.com.br

${ }^{2}$ Empresa Brasileira de Pesquisa Agropecuária - EMBRAPA, Meio-Norte, Núcleo de Pesquisa com Abelhas - NUPA, Av. Duque de Caxias, 5650, Buenos Aires, CEP 64006-220, Teresina - PI, Brasil

${ }^{*}$ A quem a correspondência deve ser enviada 
Nesse sentido, o presente trabalho teve como objetivo avaliar o perfil sensorial e a aceitabilidade de méis de M. scutellaris e $M$. quadrifasciata submetidos às técnicas de pasteurização e desumidificação, visando obter maior vida de prateleira.

\section{Material e métodos}

O experimento foi conduzido no Laboratório de Entomologia do Centro de Ciências Agrárias, Ambientais e Biológicas da Universidade Federal do Recôncavo da Bahia, em Cruz das Almas.

Foram utilizadas duas amostras de méis de M. scutellaris (Ilha de Itaparica - 1 e Costa do Sauípe - 2/Estado da Bahia) e duas amostras de méis de $M$. quadrifasciata (Tucano 1 e Serrinha 2/Estado da Bahia).

As coletas das amostras foram realizadas durante o mês de dezembro de 2005 e janeiro de 2006, período das principais floradas das regiões. O mel foi coletado com bomba de sucção e/ou seringa descartável. Foi utilizada uma seringa para cada colônia e o mel coletado foi proveniente de potes operculados, sendo armazenado em recipiente de vidro previamente esterilizado, com tampa de fecho hermético. No transporte até o laboratório, realizado no mesmo dia, os recipientes foram acondicionados em caixa de isopor com gelo. As amostras foram armazenadas sob refrigeração por aproximadamente sete dias, a uma temperatura de $8{ }^{\circ} \mathrm{C}$, em seguida foram submetidas aos processos de conservação: pasteurização com temperatura de $70^{\circ} \mathrm{C}$ por um minuto (NOGUEIRA NETO, 1997) e desumidificação em sala com desumidificador de ar com temperatura de aproximadamente $30{ }^{\circ} \mathrm{C}$ e umidade de aproximadamente $40 \%$ (MORAES et al., 1989; FONSECA et al., 2006).

As análises sensoriais foram realizadas utilizando-se como testemunha as mesmas amostras armazenadas em geladeira, técnica normalmente utilizada como meio de conservação do mel das abelhas sem ferrão pelos meliponicultores da região.

Para a análise sensorial, cada amostra foi dividida em três subamostras (testemunha, desumidificada e pasteurizada). Para compor a equipe de avaliação, foram recrutados 30 avaliadores não treinados, de ambos os sexos, com faixa etária entre 20 a 50 anos, entre os quais, alunos, funcionários e professores da Universidade Federal do Recôncavo da Bahia, em Cruz das Almas. As amostras foram servidas à temperatura ambiente, em copos plásticos, em quantidades em torno de $10 \mathrm{~g}$, codificadas com números de três dígitos, sendo a ordem de apresentação aleatória. Foram fornecidos água mineral e biscoito "água e sal" para limpeza do palato entre a avaliação das amostras (FERREIRA, 2000). Os horários dos testes foram entre as 9:00 e 12:00 horas e das 14:00 às 17:00 horas. A metodologia adotada foi a de Grosso (2006), a partir da qual foram realizadas as seguintes avaliações: fluidez, cor, aroma, cristalização, sabor e aceitabilidade (Figura 1).

Os resultados da avaliação sensorial foram submetidos à Análise de Variância (ANOVA), ao Teste de Tukey a 5\% de significância para comparação entres as médias, à Análise dos Componentes Principais (ACP) e à Análise de Cluster (pela distância euclideana média, método UPGMA - unweighted
Ficha de análise sensorial de mel

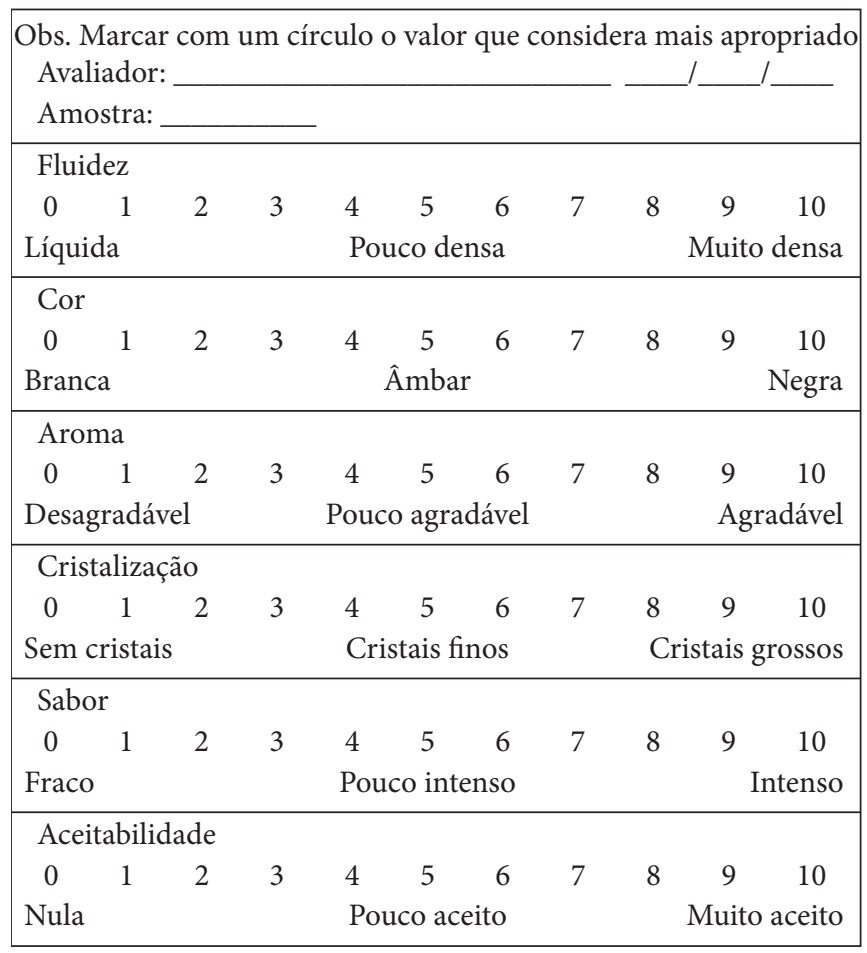

Figura 1. Ficha de avaliação sensorial para amostra de mel (GROSSO, 2006).

pair-group average). A análise do perfil sensorial das amostras foi realizada através do gráfico Aranha. Todas as análises foram realizadas utilizando-se o software SAS (1990).

\section{Resultados e discussão}

Os resultados da Análise de Variância (ANOVA) provenientes das notas atribuídas pelos provadores a cada atributo, para as amostras de méis de M. scutellaris e de M. quadrifasciata refrigerados (testemunha), pasteurizados e desumidificados avaliados sensorialmente, são apresentados na Tabela 1.

O perfil sensorial de cada um desses tratamentos é mostrado graficamente nas Figuras 2 e 3, em que o valor médio atribuído pelos provadores a cada atributo (fluidez, cor, aroma, cristalização, sabor e aceitabilidade) é marcado no eixo correspondente. O centro da figura representa o ponto zero da escala utilizada na avaliação, enquanto a intensidade aumenta do centro para a periferia. $O$ perfil sensorial se revela quando se faz a conexão dos pontos (gráfico Aranha).

Os resultados obtidos revelam que os tratamentos utilizados para a conservação do mel não interferiram nos atributos cristalização, sabor e aceitabilidade. $\mathrm{O}$ atributo aroma não apresentou diferença estatística significativa a 5\% (Teste de Tukey), quando avaliado na mesma amostra nos diferentes tratamentos. Entretanto, para este mesmo atributo foi constatada diferença apenas entre alguns tratamentos, comparando-se as diferentes amostras de mel de M. quadrifasciata 1 e 2 (Tabela 1). Com relação ao atributo cor, não houve diferença estatística entre 
Tabela 1. Média dos atributos sensoriais encontrados nas amostras de méis de M. scutellaris (Ilha de Itaparica - 1 e Costa do Sauípe - 2 / Estado da Bahia) e M. quadrifasciata (Tucano 1 e Serrinha 2 / Estado da Bahia) submetidos a processos de conservação

\begin{tabular}{|c|c|c|c|c|c|c|}
\hline \multirow[t]{2}{*}{ Espécie de abelha/tratamento } & \multicolumn{6}{|c|}{ Atributos } \\
\hline & Cristalização & Sabor & Aroma & Cor & Fluidez & Aceitabilidade \\
\hline M. scutellaris - Testemunha (1) & $0,54^{\mathrm{a}}$ & $5,00^{\mathrm{a}}$ & $6,69^{\mathrm{abc}}$ & $1,92^{\mathrm{de}}$ & $2,65^{\mathrm{bcd}}$ & $5,04^{\mathrm{a}}$ \\
\hline M. scutellaris - Desumidificada (1) & $1,23^{\mathrm{a}}$ & $6,12^{\mathrm{a}}$ & $6,92^{\mathrm{abc}}$ & $2,35^{\mathrm{cd}}$ & $7,46^{\mathrm{a}}$ & $5,08^{\mathrm{a}}$ \\
\hline M. scutellaris - Pasteurizada (1) & $0,38^{\mathrm{a}}$ & $5,15^{\mathrm{a}}$ & $5,69^{\mathrm{abc}}$ & $2,04^{\mathrm{ed}}$ & $1,92^{\mathrm{d}}$ & $5,15^{\mathrm{a}}$ \\
\hline M. scutellaris - Testemunha (2) & $0,85^{\mathrm{a}}$ & $6,27^{a}$ & $5,81^{\mathrm{abc}}$ & $3,58^{\mathrm{abc}}$ & $3,19^{\mathrm{bcd}}$ & $6,19^{\mathrm{a}}$ \\
\hline M. scutellaris - Desumidificada (2) & $1,27^{\mathrm{a}}$ & $6,08^{\mathrm{a}}$ & $6,62^{\mathrm{abc}}$ & $3,69^{\mathrm{ab}}$ & $7,12^{\mathrm{a}}$ & $5,85^{\mathrm{a}}$ \\
\hline M. scutellaris - Pasteurizada (2) & $0,85^{\mathrm{a}}$ & $6,65^{\mathrm{a}}$ & $6,00^{\mathrm{abc}}$ & $3,69^{\mathrm{ab}}$ & $2,85^{\mathrm{bcd}}$ & $7,19^{\mathrm{a}}$ \\
\hline M. quadrifasciata - Testemunha (1) & $0,77^{\mathrm{a}}$ & $5,62^{\mathrm{a}}$ & $5,96^{c}$ & $4,42^{\mathrm{a}}$ & $3,73^{\mathrm{b}}$ & $5,31^{\mathrm{a}}$ \\
\hline M. quadrifasciata - Desumidificada (1) & $1,08^{\mathrm{a}}$ & $5,62^{\mathrm{a}}$ & $5,65^{\mathrm{abc}}$ & $4,00^{\mathrm{ab}}$ & $6,85^{\mathrm{a}}$ & $5,69^{\mathrm{a}}$ \\
\hline M. quadrifasciata - Pasteurizada (1) & $1,04^{\mathrm{a}}$ & $5,92^{\mathrm{a}}$ & $5,19^{\mathrm{bc}}$ & $4,62^{\mathrm{a}}$ & $3,50^{\mathrm{bc}}$ & $5,46^{\mathrm{a}}$ \\
\hline M. quadrifasciata - Testemunha (2) & $0,46^{\mathrm{a}}$ & $6,23^{\mathrm{a}}$ & $6,54^{\mathrm{abc}}$ & $1,90^{\mathrm{d}}$ & $2,00^{\mathrm{cd}}$ & $5,88^{\mathrm{a}}$ \\
\hline M. quadrifasciata - Desumidificada (2) & $1,08^{\mathrm{a}}$ & $6,81^{\mathrm{a}}$ & $7,46^{\mathrm{a}}$ & $2,77^{\mathrm{bcd}}$ & $6,69^{\mathrm{a}}$ & $6,96^{\mathrm{a}}$ \\
\hline M. quadrifasciata - Pasteurizada (2) & $0,35^{\mathrm{a}}$ & $6,23^{\mathrm{a}}$ & $7,19^{\mathrm{ab}}$ & $1,96^{\mathrm{ed}}$ & $1,92^{\mathrm{d}}$ & $6,31^{\mathrm{a}}$ \\
\hline
\end{tabular}

Médias seguidas de mesma letra, na mesma coluna, não diferem significativamente entre si pelo Teste de Tukey $(\mathrm{p}<0,05)$.
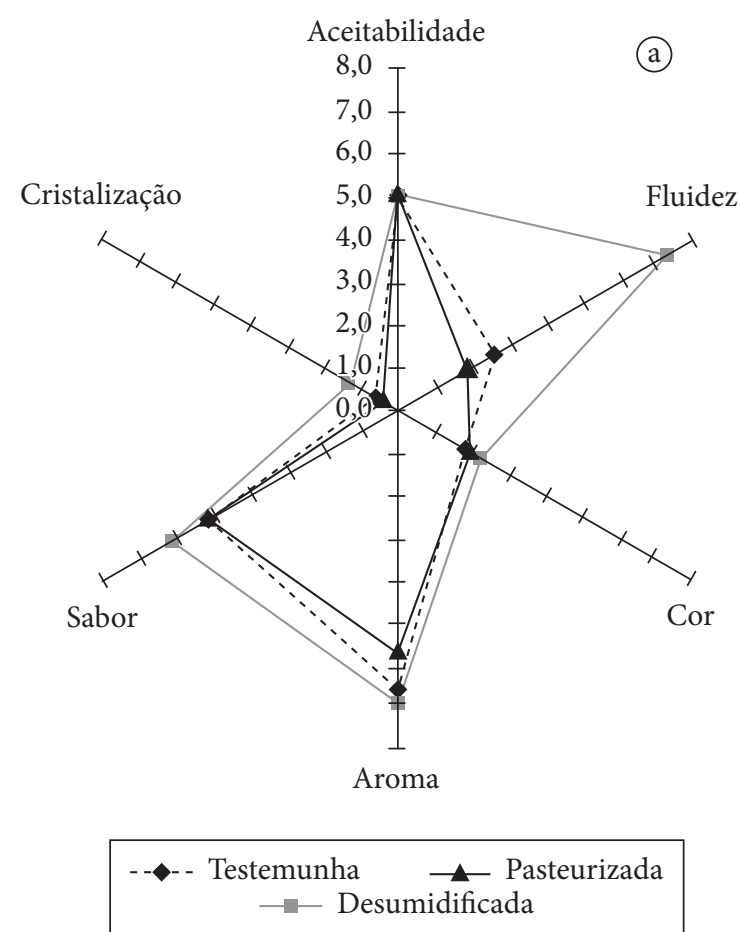
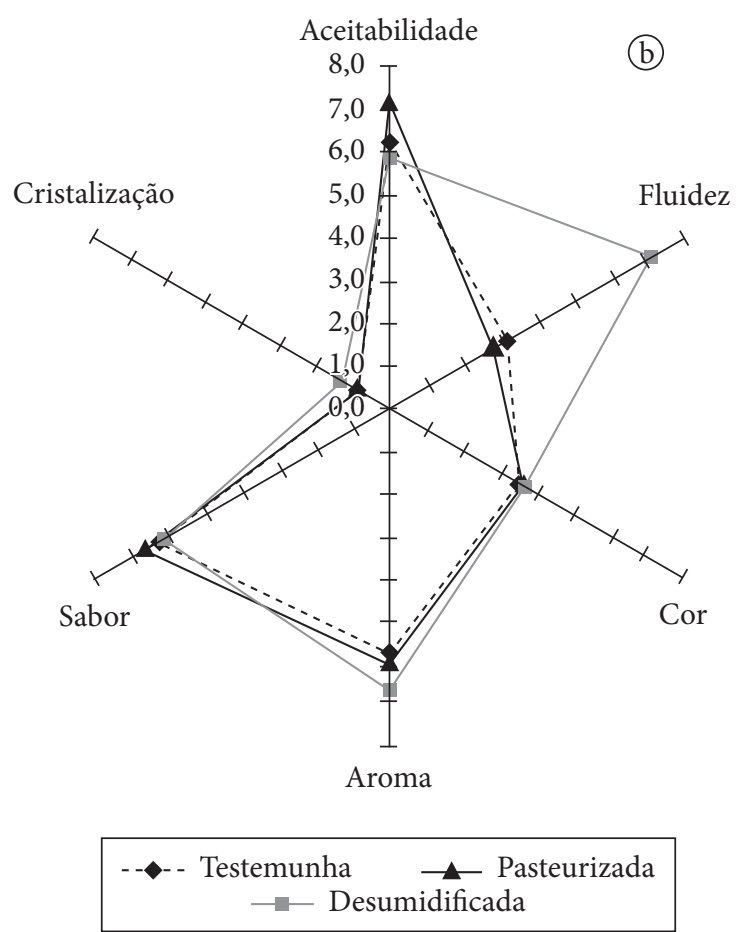

Figura 2. Perfil sensorial de amostras de méis de M. scutellaris submetidas a processos de conservação: a) Ilha de Itaparica; e b) Costa do Sauípe.

os tratamentos na mesma amostra, constatando-se diferenças apenas entre as amostras.

As diferenças apresentadas para o atributo aroma e cor podem ser explicadas pelo fato da origem do mel ser influenciada por diversos fatores. De acordo com Carvalho et al. (2006), a composição do mel pode variar em função da flora, do lugar, época de colheita, manejo e, principalmente, da espécie de abelha que o produziu.

No que diz respeito ao atributo fluidez, houve diferença estatística para o tratamento desumidificação entre o mel das duas espécies de abelhas sem ferrão. Esse resultado era esperado devido à perda de água durante a desumidificação, tornando-o mais denso, o que lhe atribui uma maior nota (Figura 1).
As similaridades e diferenças entre as amostras de méis submetidas ao tratamento de conservação são representadas nas Figuras 4 e 5 . As amostras similares ocupam as regiões próximas no gráfico e são caracterizadas pelos vetores (atributos) que se apresentam mais próximos a elas.

A Figura 4 apresenta o resultado obtido na Análise de Cluster (distância euclideana média, método UPGMA unweighted pair-group average) envolvendo a análise sensorial dos diferentes tratamentos para a conservação do mel. Foi possível observar a formação de seis grupos, identificados a seguir: grupo 1, formado por uma amostra $M$. quadrifasciata - Desumidificada (2); grupo 2, por duas amostras M. quadrifasciata - Pasteurizada (1) e M. quadrifasciata - Testemunha (1); 


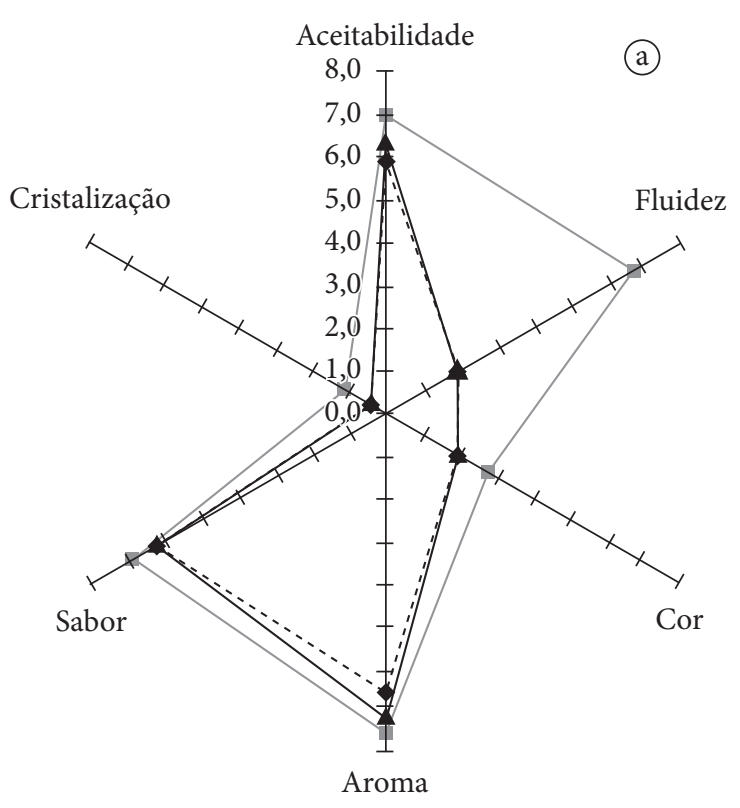

- - Testemunha $\longrightarrow$ Pasteurizada

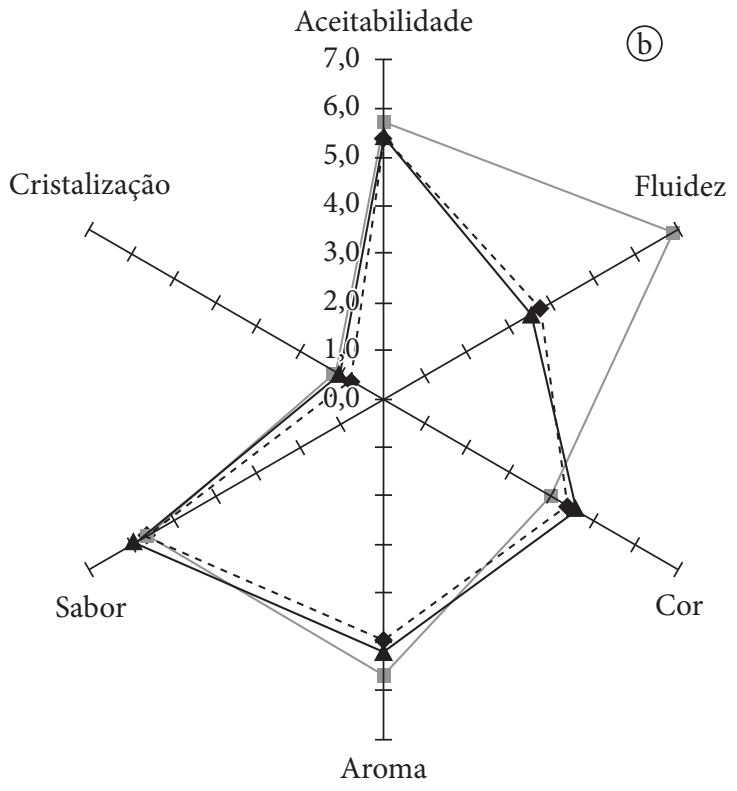

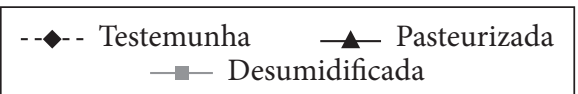

Figura 3. Perfil sensorial de amostras de méis de M. quadrifasciata submetidas a processos de conservação: a) Tucano; e b) Serrinha.

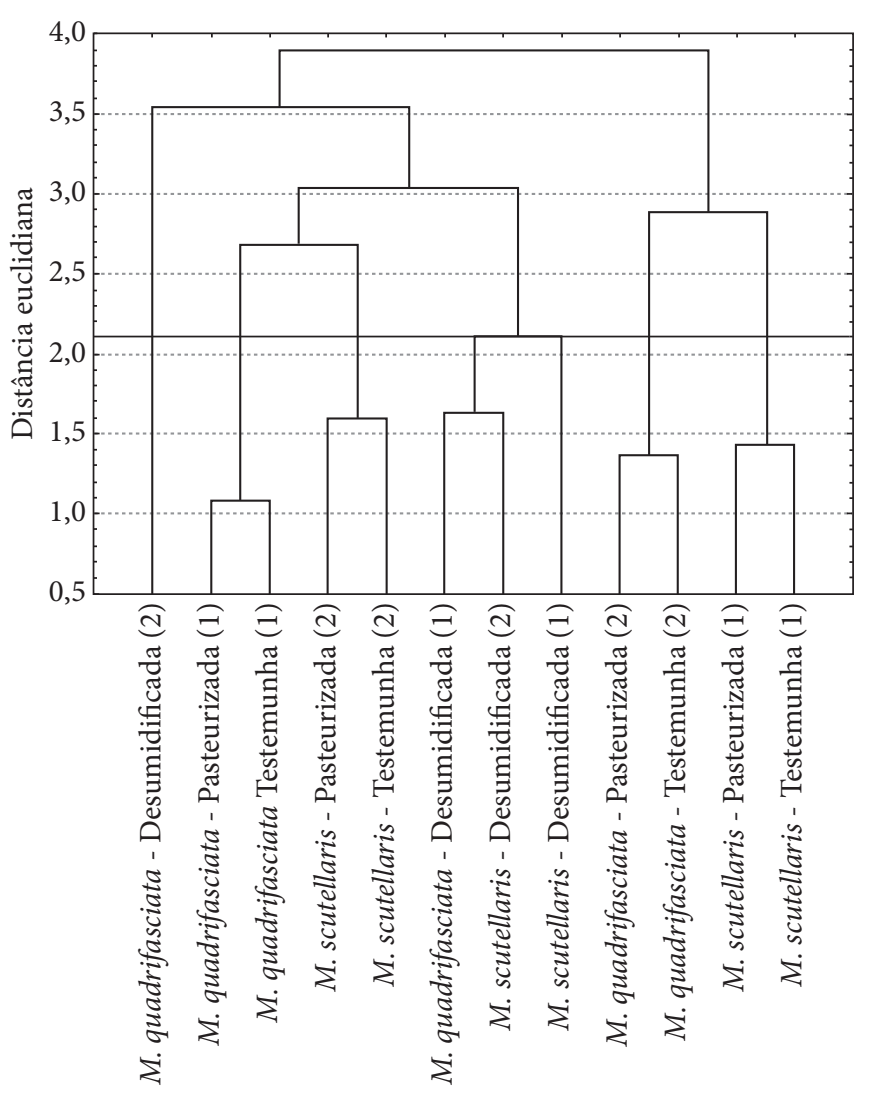

Figura 4. Fenograma obtido pela Análise de Cluster para a análise sensorial de amostras de méis de M. scutellaris e M. quadrifasciata submetidos a processos de conservação. grupo 3, por duas amostras M. scutellaris - Pasteurizada (2) e M. scutellaris - Testemunha (2); grupo 4, por três amostras M. quadrifasciata - Desumidificada (1), M. scutellaris - Desumidificada (2) e M. scutellaris - Desumidificada (1); grupo 5, por duas amostras, M. quadrifasciata - Pasteurizada (2) e M. quadrifasciata - Testemunha (2) e o grupo 6, formado por duas amostras M. scutellaris - Testemunha (1) e M. scutellaris - Pasteurizada (1). Observados os grupos formados, constatase que o grupo 1 e o 4 são formados por amostras de méis que passaram pelo processo de desumidificação, o grupo 1 se distanciou do grupo 4 provavelmente por apresentar valores mais altos para o aroma, sabor e aceitabilidade, apesar de não diferir estatisticamente entre si a 5\% de significância (Tabela 1). Essa diferença é aceitável por se tratarem de amostras diferentes.

Os demais grupos (grupos 2, 3, 5 e 6) foram formados pelas mesmas amostras nos tratamentos testemunha e pasteurizadas, mostrando similaridade entre estes tratamentos. A diferença apresentada para o tratamento desumidificada, que contribui para a formação dos grupos 1 e 4, deve-se provavelmente pelo alto valor apresentado para a fluidez destas amostras, o que as tornou mais densas (Tabela 1), essa diferença apresentada para a fluidez era esperada pela perda da umidade do mel para proporcionar ao produto uma maior vida de prateleira.

Avaliando a projeção da Figura 5 é possível visualizar a distribuição espacial das amostras avaliadas sensorialmente. Dentre os atributos sensoriais avaliados, a cristalização, no Componente Principal 1, foi quem mais contribuiu (Tabela 3 e Figuras 5 e 6). A amostra de mel de M. quadrifasciata - Pasteurizada (2) foi a que mais se distanciou no eixo $\mathrm{X}$, provavelmente por apresentar 
um menor valor para a cristalização, o que a distanciou das demais. Apesar dessa distância e da diferença entre os valores para o atributo cristalização, não apresentou diferença estatística em nível de 5\% de significância (Tabela 1).

Na ACP (Análise dos Componentes Principais) o primeiro componente contribuiu com $41,21 \%$ da variância total e o segundo com $33,81 \%$, representando os dois primeiros eixos fatoriais $(75,02 \%)$ na variância total (Tabela 2). Em uma ACP, se os dois ou os três primeiros componentes acumularem uma porcentagem relativamente alta da variação total, em geral acima de 70\%, eles explicarão satisfatoriamente a variabilidade manifestada entre as amostras avaliadas (MARDIA et al., 1979). No presente trabalho, verifica-se que a porcentagem acumulada da variação total $(75,02 \%)$ dos dois primeiros componentes explica satisfatoriamente a variabilidade nas amostras avaliadas.

Para o Componente Principal 2, o aroma (Tabela 3 e Figuras 5 e 6) foi quem mais contribuiu para a separação do grupo. A amostra de mel de M. quadrifasciata - Desumidificada (2) foi a que mais se distanciou no eixo Y, provavelmente por apresentar um maior valor para o aroma. Apesar dessa distância e da diferença entre os valores para o atributo aroma, só duas amostras diferiram estatisticamente ente si (Tabela 1).

Avaliando a Figura 5, constata-se que as amostras desumidificadas encontram-se à esquerda do eixo $\mathrm{Y}$ e mais distante do centro que as demais amostras, constatando-se um distanciamento da amostra M. quadrifasciata - Desumidificada (2); como o verificado na Análise de Cluster. Para as demais amostras também foram constatadas as mesmas proximidades apresentadas na Análise de Cluster (Figuras 4 e 5).

Avaliando o perfil sensorial e a aceitabilidade dos diferentes tratamentos utilizados para a conservação do mel das abelhas sem ferrão, constata-se que não ocorreu diferença entre eles quando avaliados na mesma amostra.

Tabela 2. Estimativas das variâncias (autovalores) e porcentagem de contribuição e de contribuição acumulada (\%) obtidas por meio de Análise de Componentes Principais (ACP), considerando os seis atributos sensoriais avaliados nas amostras de méis de M. scutellaris e M. quadrifasciata submetidos a processos de conservação.

\begin{tabular}{cccc}
\hline $\begin{array}{c}\text { Componentes } \\
\text { principais }\end{array}$ & Autovalores & $\begin{array}{c}\text { Contribuição } \\
(\%)\end{array}$ & $\begin{array}{c}\text { Contribuição } \\
\text { acumulada }(\%)\end{array}$ \\
\hline 1 & 2,47 & 41,21 & 41,21 \\
2 & 2,03 & 33,81 & 75,02 \\
\hline
\end{tabular}

Tabela 3. Contribuição para a formação do componente principal dos atributos avaliados na análise sensorial das amostras de méis de M. scutellaris e M. quadrifasciata submetidos a processos de conservação.

\begin{tabular}{lcc}
\hline Atributos analisados & \multicolumn{2}{c}{ Componentes principais } \\
\cline { 2 - 3 } & 1 & 2 \\
\hline Aceitabilidade & 0,120285 & 0,169028 \\
Fluidez & 0,240932 & 0,027111 \\
Cor & 0,100320 & 0,255983 \\
Aroma & 0,017454 & 0,334876 \\
Sabor & 0,217638 & 0,137736 \\
Cristalização & 0,303372 & 0,075265 \\
\hline
\end{tabular}

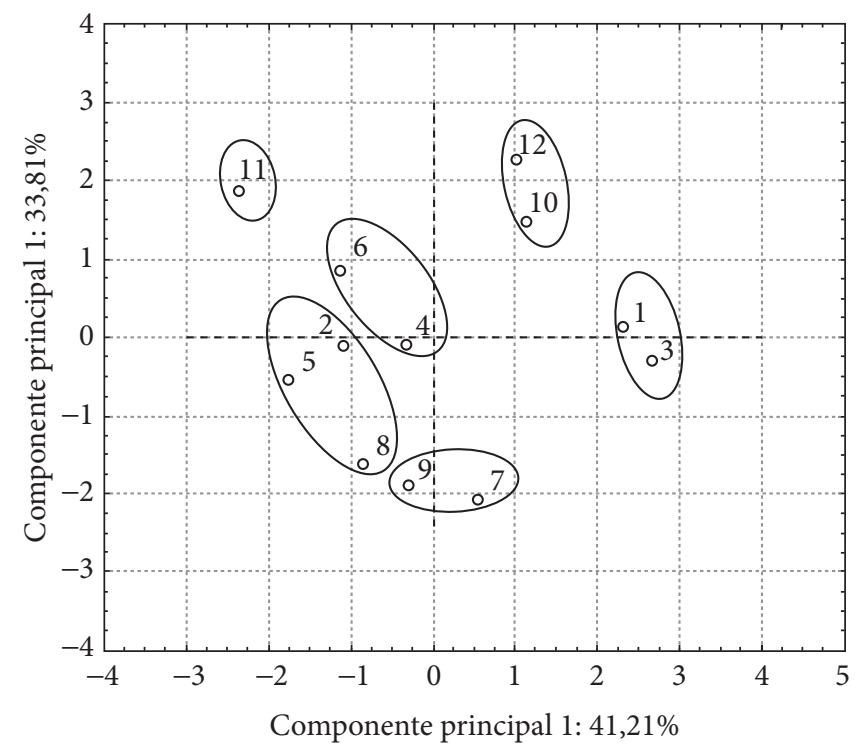

Figura 5. Projeção da ACP dos tratamentos utilizados na análise sensorial de amostras de méis de $M$. scutellaris (Ms) (Ilha de Itaparica - 1 e Costa do Sauípe - 2 / Estado da Bahia) e M. quadrifasciata (Mq) (Tucano 1 e Serrinha 2 / Estado da Bahia) submetidos a processos de conservação: 1) $M s$ - Testemunha (1); 2) $M s$ - Desumidificada (1); 3) $M s$ - Pasteurizada (1); 4) $M s$ - Testemunha (2); 5) $M s$ - Desumidificada (2); 6) $M s$ - Pasteurizada (2): 7) $M q$ - Testemunha (1); 8) $M q$ Desumidificada (1); 9) $M q$ - Pasteurizada (1); 10) $M q$ - Testemunha (2); 11) $M q$ - Desumidificada (2); e 12) $M q$ - Pasteurizada (2).

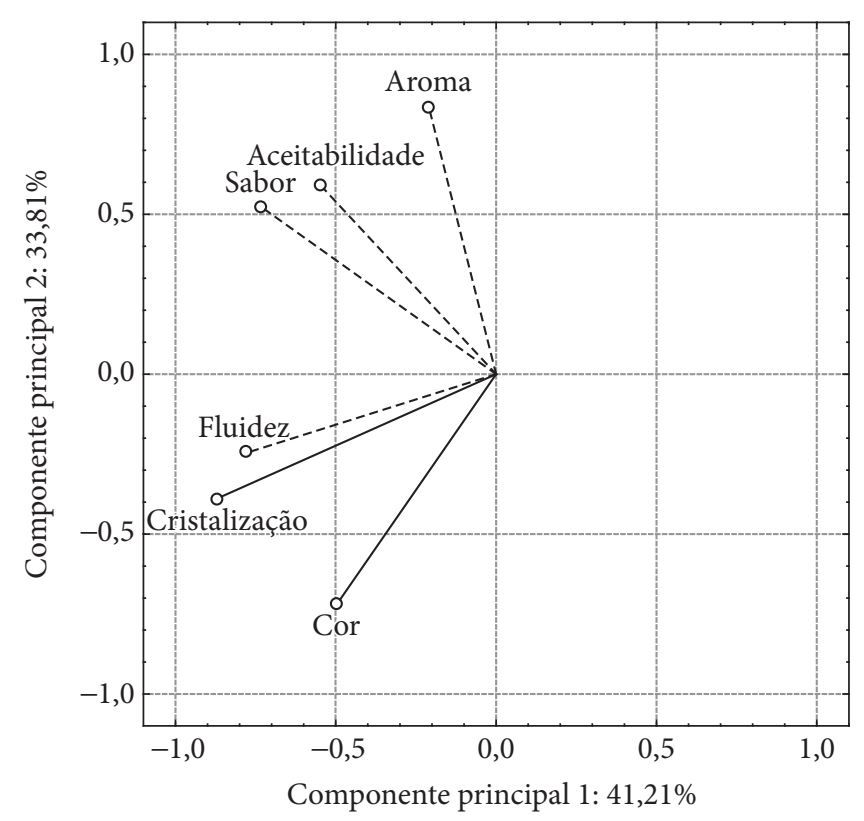

Figura 6. Projeção da ACP dos atributos sensoriais avaliados nas amostras de méis de M. scutellaris e M. quadrifasciata submetidos a processos de conservação. 
Embora a conservação do mel por meio da pasteurização seja uma técnica importante, por reduzir riscos causados por alimentos líquidos e por evitar a fermentação do mel (NOGUEIRA NETO, 1997), após a abertura do recipiente contendo mel é necessário conservá-lo sob refrigeração para evitar processos de deterioração. Considerando esse aspecto, a desumidificação é mais vantajosa como técnica de conservação do mel das abelhas sem ferrão, uma vez que é possível armazená-lo com o teor adequado de umidade, evitando a sua fermentação e garantindo maior durabilidade.

\section{Conclusões}

Os méis das abelhas sem ferrão submetidos à pasteurização e desumidificação apresentam boa aceitabilidade.

O processo de desumidificação parece ser mais vantajoso para a conservação do mel das abelhas sem ferrão, por permitir uma maior vida de prateleira.

\section{Agradecimentos}

À Fundação de Amparo à Pesquisa do Estado da Bahia (FAPESB), pela concessão da Bolsa de Pós-Doutorado para a autora Geni da Silva Sodré, e ao Conselho Nacional de Desenvolvimento Científico e Tecnológico (CNPq), pela concessão da Bolsa de Produtividade em Pesquisa ao coautor Carlos Alfredo Lopes de Carvalho.

\section{Referências bibliográficas}

ARAÚJO, A. H. et al. Análise sensorial de água de coco in natura em comparação à pasteurizada. In: CONGRESSO BRASILEIRO DE CIÊNCIA E TECNOLOGIA DE ALIMENTOS, 17. Fortaleza, 2000.

Anais... Fortaleza: Sociedade Brasileira de Ciência e Tecnologia de Alimentos, 2000. v.1, p. 3.44 .
CARDELLO, H. M. A. B.; CARDELLO, L. Teor de vitamina C, atividade de ascorbato oxidase e perfil sensorial de manga (Mangífera índica L.) var. haden, durante o amadurecimento. Ciência Tecnologia de Alimento, v.18, n. 2, p. 211-217, 1998.

CARVALHO, C. A. L. et al. Perfil sensorial de amostras de méis de espécies de abelhas sem ferrão do Estado da Bahia. Magistra, v. 18, n. 4, p. 265-269, 2006.

CARVALHO, C. A. L. et al. Mel de Abelha sem ferrão: contribuição para a caracterização físico-química. Cruz das Almas: Nova Civilização, 2005. 32p. (Serie Meliponicultura No 04).

FERREIRA, V. L. P. (Coord.). Análise sensorial - Testes discriminativos e afetivos. Campinas: Sociedade Brasileira de Ciência e Tecnologia de Alimentos, 2000. 77p. (Manual Série Qualidade).

FONSECA, A. A. O. et al. Qualidade do mel de abelhas sem ferrão: uma proposta para boas práticas de fabricação. Cruz das Almas: Nova Civilização, 2006. 70 p. (Serie Meliponicultura No 05).

FRÍAS, I.; HARDISSON, A. Estudio de los parámetros analíticos de interés en la miel. II: Azúcares, cenizas y contenido mineral y color. Alimentaria, v. 28, n. 235, p. 41-43, 1992.

GROSSO, G. S. Criterios relativos al análisis sensorial de mieles. Apiservices-Galerie Virtuelle Apicole. França, 2006. Disponível em: <http://www.beekeeping.com/articulos/salamanca/index.htm $>$. Acesso em: 3 fev. 2006.

MARDIA, L. V.; KENI, J. T.; BIBBY, J. M. Multivariate analysis. London: Academic Press, 1979. 521 p.

MORAES, R. M.; BENEVIDES, L. H. T. S.; MENEZES, A. A desumidificação no mel no Brasil. Apicultura \& Polinização, n. 13, p. 27-29, 1989.

NOGUEIRA-NETO, P. Vida e criação de abelhas indígenas sem ferrão. São Paulo: Nogueirapis. 1997. 445 p.

PIANA, M. L. et al. Sensory analysis applied to honey: state of the art. Apidologie, v. 35, p. 26-37. 2004.

SILVA, J. A. Tópicos de tecnologia de alimentos. São Paulo: Varela, 2000. 227 p.

STATISTICAL ANALISYS SISTEM.. SAS/STAT: User's guide. [S. I.] SAS Institute Inc., 1990. (Version 6.4). 